Research Paper

\title{
High preoperative serum globulin in hepatocellular carcinoma is a risk factor for poor survival
}

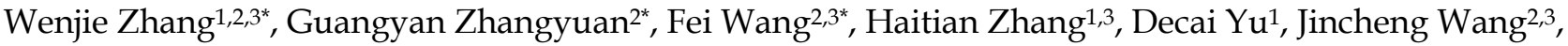 \\ Kangpeng Jin ${ }^{1,3}$, Weiwei Yu ${ }^{1,2}$, Yang Liu1,3, Beicheng Sun ${ }^{2,3} \bowtie$ \\ 1. Department of Hepatobiliary Surgery, The Affiliated Drum Tower Hospital of Nanjing University Medical School, Nanjing 210029, Jiangsu Province, \\ P.R.China \\ 2. Department of Hepatobiliary Surgery of Drum Tower Clinical Medical College, Nanjing Medical University, Nanjing, China. \\ 3. Liver Transplantation Center, The First Affiliated Hospital of Nanjing Medical University, Nanjing, Jiangsu Province, P.R.China. \\ *The authors contributed equally to this work \\ $\triangle$ Corresponding author: sunbc@nju.edu.cn (B.S.). Telephone: 86-25-68136746; Fax: 86-25-86560946 \\ (c) Ivyspring International Publisher. This is an open access article distributed under the terms of the Creative Commons Attribution (CC BY-NC) license \\ (https:// creativecommons.org/licenses/by-nc/4.0/). See http://ivyspring.com/terms for full terms and conditions.
}

Received: 2018.08.27; Accepted: 2019.05.19; Published: 2019.06.09

\begin{abstract}
Background: Serum globulin (GLB), albumin (ALB) and albumin/globulin ratio (AGR) have been reported as prognosis related factors for certain malignancies; however, the prognostic value of globulin (GLB) in hepatocellular carcinoma (HCC) has rarely been studied. This study was performed to evaluate whether GLB analysis could be applied for the prediction of the prognosis of patients received liver resection.

Methods: A training cohort study involving $210 \mathrm{HCC}$ patients undergoing curative liver resection between January 2007 and December 2012, and a validation cohort involving $100 \mathrm{HCC}$ patients contemporaneously undergoing curative liver resection in another set were recruited. The survival curves were graphed and log-rank test was performed to analyze the differences between the curves. The cutoff value was selected by $\mathrm{X}$-title program.

Results: Univariate and multivariate analysis indicated that high serum GLB level is a risk factor for poor cancer-specific survival (CSS) $(P<0.05)$. Conversely, high ALB level is a prediction for favor CSS $(P=0.010)$.

Conclusions: We identified the preoperative high GLB level as a prognostic risk factor for patients after treatment of liver cancer resection. This easily obtained variable may act as an available clinical biomarker to predict the prognosis of such patients.
\end{abstract}

Key words: HCC, globulin, albumin, survival

\section{Introduction}

Hepatocellular carcinoma (HCC) ranks $5^{\text {th }}$ (men) and $9^{\text {th }}$ (women) in frequency and is globally third leading cause of cancer-related death [1]. Although most cases of HCC occur in Africa and Eastern Asia, trends in HCC have shown considerable increases in low-incidence areas such as the United States and Canada [2]. Chronic hepatitis B or C virus (HBV or $\mathrm{HCV}$ ) infections are the major risk factors for HCC [3]. The increasing level of diagnosis in high-risk populations, such as ultrasonography and computed tomographic scanning, has led to the identification of increasing numbers of patients with HCC. Although the survival benefit of surgical techniques and preoperative management in HCC has been well made, its prognosis remains dismal [4, 5]. Actually, serum alpha-fetoprotein (AFP) was widely recognized and utilized as a diagnostic and prognostic marker of HCC $[6,7]$.However, AFP was increased in $211-58 \%$ of patients with chronic hepatitis or cirrhosis without HCC, and $30-40 \%$ of HCC patients were AFP negative $[6,8,9]$. Moreover, AFP was also reported to have no prognostic role in small 
hepatocellular carcinoma patients with well-compensated cirrhosis [10]. Thus, identifying simple and reliable biological markers to predict patient who are at high-risk for early death and recurrence would be important.

Albumin (ALB) and globulin (GLB), the two major constituents of serum proteins, are routinely measured by biochemical examinations. Both of them are considered to play a pivotal role in the inflammatory process. Serum ALB level, as well as albumin/globulin ratio (AGR), has been known as a prognostic indicator in several types of cancer, including gastric cancer, colorectal cancer, breast cancer, ovarian cancer and nasopharyngeal carcinoma [11-13]. In contrast to the considerable amount of researches on ALB and AGR, however, whether the impact of the GLB and $A / G$ ratio is associated with outcome in patients with HCC has not yet been elucidated. Therefore, the purpose of this study was to assess whether preoperative globulin and A/G ratio had a prognostic value in patients with HCC.

\section{Methods}

\section{Study population}

Data involved was collected from patients suffering HCC and receiving liver resection in the First Affiliated Hospital of Nanjing Medical University (NJMU) and Nanjing Drum Tower Hospital, Nanjing, China between January 2007 and December 2012. The latest detection of serum ALB level, GLB level and albumin/globulin ratio (AGR) was recorded before liver resection operation. Patients aged between 18 and 85 years at diagnosis, undergoing partial liver resection with pathologically confirmed HCC as primary tumor were included. Patients who had preexisting diseases of immune system, received immunosuppressive therapies involving recent exposure of steroid or other immunity medicine before operation, and those died within 30 days after surgery were excluded from this study. Meanwhile, patients with incomplete clinical information including vascular invasion, tumor multiplicity, tumor size, Edmondson grade, or without follow up were excluded.

The study was approved by the Institutional Ethics Committee of the First Affiliated Hospital of NJMU. And our research was performed according to the Helsinki Declaration and government policies. Written informed consent was obtained from all patients.

\section{Statistical analysis}

Basic information about patients, and data of clinicopathological and laboratory examination was retrieved from the medical recording system of the first affiliated hospital of NJMU. Patients' clinical features including sex, age, level of $\mathrm{HbsAg}$, ALT (U/L), AFP (ng/ml), GLB (g/L), ALB (g/L), cirrhosis or not, pathological features about vascular invasion, tumor multiplicity, tumor size, Edmondson grade and information on follow up was obtained. Serum level of GLB, ALB as well as HbsAg, ALT and AFP was detected by an automatic biochemical analyzer (Hitachi 7600). All the patients were further divided into two groups based on criterion as follows: (1) sex: male or female; (2) age: $\leq 60$ years (young group) or $>$ 60 years (old group); (3) serum HbsAg: negative (normal) or positive (abnormal); (4) ALT level: $\leq 45$ $\mathrm{U} / \mathrm{L}$ (normal) or $>45 \mathrm{U} / \mathrm{L}$ (abnormal); (5) AFP level: $\leq 13.6 \mathrm{U} / \mathrm{L}$ (normal) or $>13.6 \mathrm{U} / \mathrm{L}$ (abnormal); (6) cirrhosis in liver tissues: absent or present; (7) tumor vascular invasion: absent or present; (8) tumor multiplicity: solitary or multiple; (9) tumor size: $\leq 5$ $\mathrm{cm}$ or $>5 \mathrm{~cm}$; (10) Edmondson grade: I-II or III-IV. The AGR value was calculated, [AGR = Albumin/(Total protein - Albumin)]. The cutoff value was selected by X-title program and set as follows: GLB, $32.70 \mathrm{~g} / \mathrm{L}$; ALB, $40.60 \mathrm{~g} / \mathrm{L}$; AGR, 1.40. CSS, calculated from the date of diagnosis to the date of cancer specific death was selected as the primary endpoint of the study. Deaths were set as events and deaths attributed to other causes were set as censored observation. Kaplan-Meier estimates were applied to graph the survival curves, and log-rank test was performed to analyze the differences between the curves. Risk factors for survival outcomes in HCC patients were analyzed using Multivariable Cox regression models. Chi-square test was performed for categorical variables. 5-year CSS was evaluated from Kaplan-Meier curves. SPSS 17.0 for Windows (IBM Corp, Armonk, NY, USA) was used to perform all the statistical analyses. $\mathrm{P}<0.05$ (two-tailed test) was considered statistical significant.

\section{Results}

\section{Patients}

210 patients in total initially diagnosed as HCC and receiving liver resection in the first affiliated hospital of NJMU from 2007 to 2012 were involved in this study as a training cohort (Table 1). Among them, 173 are male $(82.4 \%)$ and $37(17.6)$ are female. The average age of the patients was 55 years (range, 47-75 years). Of these $184(87.6 \%)$ were chronic hepatitis B patients, and $160(76.2 \%)$ were diagnosed as liver cirrhosis. During follow-up, 107 patients experienced HCC recurrence and 75 patients developed tumor metastasis. By the end of follow-up, 153/210 patients $(72.9 \%)$ died of HCC. 

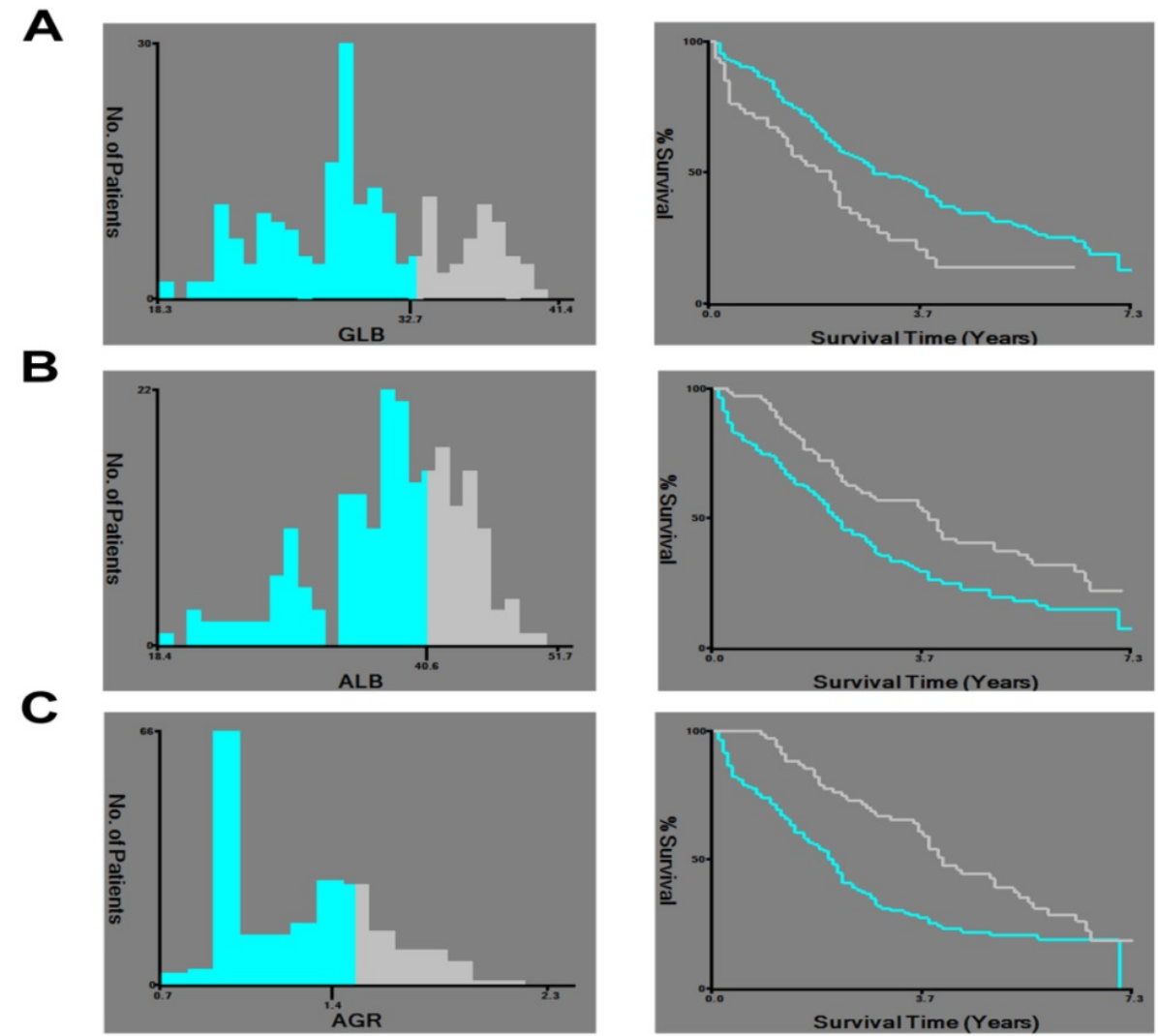

Figure 1. X-tile analysis of survival data of HCC patients. X-tile analysis was done on patient data from our center, equally divided into training and validation sets. $X$-tile plots of the training sets are shown in the left panels, with plots of matched validation sets shown in the smaller inset. The optimal cut-point highlighted by the black circle in the left panels is shown on a histogram of the entire cohort (middle panels), and a Kaplan-Meier plot (right panels). $P$ values were determined using the cutoff point defined in the training set and applying it to the validation set. A: Shows the optimal cutoff point for the GLB $(32.70, X 2=10.625, P=0.001)$. B: Shows the optimal cutoff point for the ALB (40.60, X2 $=10.038, P=0.002)$. C: Shows the optimal cutoff point for the AGR $(1.40, X 2=13.172, P<0.001)$.

Table 1. Clinical features of patients with HCC in the training cohort

\begin{tabular}{lll}
\hline & $\mathrm{n}=210$ & $\%$ \\
\hline $\begin{array}{l}\text { Age (year) } \\
\text { Sex }\end{array}$ & $55(47-75)$ & \\
male & 173 & $82.4 \%$ \\
female & 37 & $17.6 \%$ \\
HbsAg & & \\
negative & 26 & $12.4 \%$ \\
positive & 184 & $87.6 \%$ \\
ALT (U/L) & & \\
$\leq 45$ & 159 & $75.7 \%$ \\
$>45$ & 51 & $24.3 \%$ \\
AFP (ng/ml) & & \\
$\leq 13.6$ & 79 & $37.6 \%$ \\
$>13.6$ & 131 & $62.4 \%$ \\
Cirrhosis & & \\
absent & 50 & $23.8 \%$ \\
present & 160 & $76.2 \%$ \\
Vascular invasion & & \\
absent & 52 & $24.8 \%$ \\
present & 158 & $75.2 \%$ \\
Tumor multiplicity & & \\
solitary & 157 & $74.8 \%$ \\
multiple & 53 & $25.2 \%$ \\
Tumor size (cm) & & $59.5 \%$ \\
$\leq 5$ & 125 & $43.8 \%$ \\
$>5$ & 85 & \\
Edmondson grade & & \\
I-II & & \\
III-IV & & \\
& & \\
\hline
\end{tabular}

\section{Identification of optimal cut-off value for GLB, ALB, and AGR}

The mean value of GLB and ALB involved were $29.88 \mathrm{~g} / \mathrm{L}$ (range, 18.30-41.40 g/L) and $37.71 \mathrm{~g} / \mathrm{L}$ (range, 18.40-51.70 g/L), respectively. And the mean value of AGR involved was 1.30 (range, 0.70-2.30). $X$-tile program was applied to set the optimal cut-off points for GLB, ALB and AGR. The GLB cutoff value for CSS was $32.70 \mathrm{~g} / \mathrm{L}$ with maximum $\chi^{2}$ log-rank value of $10.625(\mathrm{P}=0.001)$, and all patients were divided into either high $(>32.70 \mathrm{~g} / \mathrm{L})$ or low $(\leq 32.70$ g/L) GLB groups. Similarly, an ALB cutoff point of $40.60 \mathrm{~g} / \mathrm{L}$ and an AGR cutoff point of 1.40 were selected as the optimal cutoff value for survival analyses $\left(\chi^{2}=10.038, \mathrm{P}=0.002\right.$, and $\chi^{2}=13.172, \mathrm{P}<$ 0.001 , respectively) to divide the patients involved into low and high risk subsets in terms of CSS (Figure. $1)$.

\section{Association of ALB and GLB with the clinicopathological features of HCC}

On the basis of the optimal cutoff value, 138/210 patients $(65.7 \%)$ had a low ALB level and 149/210 patients $(71.0 \%)$ had a low GLB level. When stratified by the test results of serum level of AFP and 
pathological results of cirrhosis, tumor size and Edmondson grade, the patient distribution of the GLB level differed significantly. Comparing with patients in higher GLB group (12/61, 19.7\%), more patients were with low AFP level in the low GLB group $(67 / 149,43.0 \%) \quad(P=0.001)$. Conversely, significantly more patients had basic liver cirrhosis in high GLB group (52/61, 85.2 \%) than in low GLB group $(108 / 149,72.5 \%)(\mathrm{P}=0.049)$. Moreover, there were higher percentage of patients with tumor size of $\leq 5$ in the low GLB (104/149, 69.8\%) group than patients in higher GLB group $(21 / 61,34.4 \%)(\mathrm{P}=0.001)$. More patients were classified as Edmondson grade I-II in high GLB group $(29 / 122,23.8 \%)$ than in low GLB group $(7 / 64,10.9 \%)(\mathrm{P}=0.025)$.

The patient distribution of ALB level showed significant difference when stratified by the test results of HbsAg, ALT, AFP and pathological results of cirrhosis and Edmondson grade. Significantly more patients were with low HbsAg level in the high ALB group $(24 / 72,33.3 \%)$ than in the low ALB group $(2 / 138,1.4 \%)(\mathrm{P}<0.001)$. More patients were in high level of ALT in the high ALB group (61/72, 84.7\%) than in the low ALB group (98/138, 71.0\%) $(\mathrm{P}=0.028)$. And there were higher percentage of patients with a higher serum value of AFP in the high ALB group $(43 / 72,59.7 \%)$ than in the low ALB group (36/138, $26.1 \%) \quad(\mathrm{P}<0.001)$. In addition, comparing with patients in low ALB group (25/138, 18.1\%), more patients had basic liver cirrhosis in the high ALB group $(25 / 72,34.7 \%)(\mathrm{P}=0.007)$. More patients were classified as Edmondson grade I-II in high ALB group $(56 / 72,77.8 \%)$ than in low ALB group $(62 / 138,44.9 \%)$ $(\mathrm{P}<0.001)$ (Table 2).

\section{Prognostic value of ALB, GLB, and AGR}

As the results of univariate analysis indicated, the high serum of AFP $(\mathrm{P}=0.005)$ and GLB $(\mathrm{P}=0.001)$, low serum level of ALB ( $P=0.002)$, low AGR value $(\mathrm{P}<0.001)$ and other clinicopathological factors involving tumor vascular invasion $(\mathrm{P}=0.012)$, tumor multiplicity $(\mathrm{P}=0.001)$, tumor size $(\mathrm{P}<0.001)$ and Edmondson grade $(\mathrm{P}<0.001)$ were significant risk factors for poor survival of HCC patients suffering liver resection (Table 3).

Various prognostic factors were adjusted with the performance of multivariate Cox regression analysis. In accordance with the results of univariate analysis, the high serum level of GLB (hazard ratio [HR] 1.865; $95 \%$ confidence interval [CI] 1.089-3.194, $\mathrm{P}=0.023$ ), low serum level of ALB (HR 0.658; $95 \% \mathrm{CI}$ 0.436-0.993, $\mathrm{P}=0.046)$, tumor vascular invasion (HR 1.847; $95 \%$ CI 1.221-2.795, $\mathrm{P}=0.004)$, tumor multiplicity (HR 0.361; $95 \%$ CI 0.230-0.567, P<0.001), tumor size (HR 2.308; 95 \% CI 1.588-3.354, P<0.001) and Edmondson grade (HR 2.784; 95 \% CI 1.694-4.573, $\mathrm{P}<0.001)$ were suggested as independent predictive factors for HCC. A serum value of higher GLB and low ALB demonstrated a negative effect on CSS. However, the serum level of AFP and AGR score were not significant predictive factors in multivariate analysis (Table 3).

Table 2. Association among GLB, ALB and the clinical features in HCC patients.

\begin{tabular}{|c|c|c|c|c|c|c|}
\hline \multirow[t]{2}{*}{ Variable } & \multicolumn{2}{|c|}{ GLB level $(\mathrm{g} / \mathrm{L})$} & \multirow[t]{2}{*}{$\mathrm{P}$} & \multicolumn{2}{|c|}{ ALB level(g/L) } & \multirow[t]{2}{*}{$\mathrm{P}$} \\
\hline & low & high & & low & high & \\
\hline Sex & & & 0.617 & & & 0.100 \\
\hline Male & 124 & 49 & & 118 & 55 & \\
\hline Female & 25 & 12 & & 20 & 17 & \\
\hline Age & & & 0.812 & & & 0.614 \\
\hline$\leq 60$ & 110 & 46 & & 101 & 55 & \\
\hline$>60$ & 39 & 15 & & 37 & 17 & \\
\hline HbsAg & & & 0.799 & & & 0.000 \\
\hline negative & 19 & 7 & & 2 & 24 & \\
\hline positive & 130 & 54 & & 136 & 48 & \\
\hline $\operatorname{ALT}(\mathrm{U} / \mathrm{L})$ & & & 0.948 & & & 0.028 \\
\hline$\leq 45$ & 113 & 46 & & 98 & 61 & \\
\hline$>45$ & 36 & 15 & & 40 & 11 & \\
\hline $\operatorname{AFP}(\mathrm{ng} / \mathrm{ml})$ & & & 0.001 & & & 0.000 \\
\hline$\leq 13.6$ & 67 & 12 & & 36 & 43 & \\
\hline$>13.6$ & 82 & 49 & & 102 & 29 & \\
\hline Cirrhosis & & & 0.049 & & & 0.007 \\
\hline absent & 41 & 9 & & 25 & 25 & \\
\hline present & 108 & 52 & & 113 & 47 & \\
\hline Vascular invasion & & & 0.148 & & & 0.954 \\
\hline absent & 41 & 11 & & 34 & 18 & \\
\hline present & 108 & 50 & & 104 & 54 & \\
\hline Tumor multiplicity & & & 0.107 & & & 0.782 \\
\hline solitary & 116 & 41 & & 104 & 53 & \\
\hline multiple & 33 & 20 & & 34 & 19 & \\
\hline Tumor size $(\mathrm{mm})$ & & & 0.000 & & & 0.526 \\
\hline$\leq 5$ & 104 & 45 & & 80 & 45 & \\
\hline$>5$ & 21 & 40 & & 58 & 27 & \\
\hline Edmondson grade & & & 0.000 & & & 0.000 \\
\hline I-II & 109 & 9 & & 62 & 56 & \\
\hline III-IV & 31 & 61 & & 76 & 16 & \\
\hline
\end{tabular}

\section{Validation of the prognostic value of GLB}

To verify the prognostic value of GLB in HCC patients, we recruited validation cohort involving 100 HCC patients from the other cohort. Clinical information of patients was listed in Table. S1. According the cut-off we identified in Figure 1, patients were divided into two groups. As expected, high serum level of GLB (hazard ratio [HR] 1.376; 95 $\%$ confidence interval $[\mathrm{CI}]$ 1.049-2.795, $\mathrm{P}=0.032$ ), low serum level of ALB (HR 0.622; 95 \% CI 0.439-0.972, $\mathrm{P}=0.039$ ) were associated with poor outcome of HCC (Figure 2 and Table S2).

\section{Discussion}

As a kind of malignant tumor with high morbidity and motility, HCC is always characterized by delayed diagnosis and poor prognosis.[14] Treatments for HCC include open surgical operation, radiotherapy, radiofrequency ablation and 
chemotherapy.[15-17] For HCC patients at early stages without distant metastasis, surgical resection is the best choice.[18, 19] The reported five-year cancer specific survival (CSS) for HCC patients receiving liver resection is about $20 \sim 40 \%$, which is far from satisfactory. [20] The prognosis of HCC may be decided by many factors, such as age, gender, tumor size, tumor stage, vascular invasion, tumor multiplicity and so forth. [21, 22] These factors may act as predicators for the prognosis of patients after HCC resection. However, sole factor is not sufficient to make an accurate prediction, as many host and tumor related factors must be considered.

Table 3. Univariate and multivariate survival analyses evaluating GLB, ALB, and AGR influencing CSS in HCC of the training cohort.

\begin{tabular}{|c|c|c|c|c|c|}
\hline & & Univariate analy & sis & Multivariate analys & \\
\hline Variable & 5-year CCS & Log rank $\chi^{2}$ test & $\mathrm{P}$ & $\mathrm{HR}(95 \% \mathrm{CI})$ & $\mathrm{P}$ \\
\hline Sex & & 0.463 & 0.496 & & $\mathrm{NI}$ \\
\hline Male & $26.5 \%$ & & & & \\
\hline Female & $29.1 \%$ & & & & \\
\hline Age & & 0.059 & 0.808 & & NI \\
\hline$\leq 60$ & $26.6 \%$ & & & & \\
\hline$>60$ & $27.4 \%$ & & & & \\
\hline HbsAg & & 0.027 & 0.870 & & NI \\
\hline negative & $28.8 \%$ & & & & \\
\hline positive & $28.0 \%$ & & & & \\
\hline $\operatorname{ALT}(\mathrm{U} / \mathrm{L})$ & & 0.873 & 0.350 & & NI \\
\hline$\leq 45$ & $28.2 \%$ & & & & \\
\hline$>45$ & $22.1 \%$ & & & & \\
\hline $\operatorname{AFP}(\mathrm{ng} / \mathrm{ml})$ & & 7.727 & 0.005 & & 0.288 \\
\hline$\leq 13.6$ & $36.5 \%$ & & & Reference & \\
\hline$>13.6$ & $20.7 \%$ & & & $1.221(0.845-1.764)$ & \\
\hline Cirrhosis & & 3.400 & 0.065 & & NI \\
\hline absent & $30.5 \%$ & & & & \\
\hline present & $25.9 \%$ & & & & \\
\hline Vascular invasion & & 6.255 & 0.012 & & 0.004 \\
\hline absent & $27.6 \%$ & & & Reference & \\
\hline present & $25.1 \%$ & & & 1.847 (1.221-2.795) & \\
\hline Tumor multiplicity & & 11.811 & 0.001 & & 0.000 \\
\hline solitary & $18.2 \%$ & & & Reference & \\
\hline multiple & $46.2 \%$ & & & $0.361(0.230-0.567)$ & \\
\hline Tumor size (mm) & & 34.515 & 0.000 & & 0.000 \\
\hline
\end{tabular}

\begin{tabular}{|c|c|c|c|c|c|}
\hline & & Univariate analy & & Multivariate analys & \\
\hline Variable & 5-year CCS & Log rank $\chi^{2}$ test & $\mathrm{P}$ & $\mathrm{HR}(95 \% \mathrm{CI})$ & $\mathrm{P}$ \\
\hline$\leq 5$ & $34.7 \%$ & & & Reference & \\
\hline$>5$ & $14.5 \%$ & & & $2.308(1.588-3.354)$ & \\
\hline Edmondson grade & & 27.857 & 0.000 & & 0.000 \\
\hline I-II & $36.6 \%$ & & & Reference & \\
\hline III-IV & $11.4 \%$ & & & $2.784(1.694-4.573)$ & \\
\hline GLB (g/L) & & 10.625 & 0.001 & & 0.023 \\
\hline$\leq 32.7$ & $31.3 \%$ & & & Reference & \\
\hline$>32.7$ & $14.2 \%$ & & & $1.865(1.089-3.194)$ & \\
\hline $\operatorname{ALB}(g / L)$ & & 10.038 & 0.002 & & 0.046 \\
\hline$\leq 40.6$ & $20.1 \%$ & & & Reference & \\
\hline$>40.6$ & $37.7 \%$ & & & $0.658(0.436-0.993)$ & \\
\hline AGR & & 13.172 & 0.000 & & 0.358 \\
\hline$\leq 1.4$ & $20.7 \%$ & & & Reference & \\
\hline$>1.4$ & $39.4 \%$ & & & $0.811(0.519-1.267)$ & \\
\hline
\end{tabular}

NI: not included in multivariate survival analysis. HR: hazard ratio, CI: confidence interval, GLB: globulin, ALB: albumin, AGR: albumin/globulin ratio.

Globulin, including the gamma globulins or antibodies and glycoprotein, is one the most important groups of blood proteins. [23] GLB may act as a regulator in the circulatory system by assisting the blood in clotting, transporting proteins through the lipoproteins, indicating antibody levels, and so forth. High globulin levels may be attributed to chronic inflammatory diseases such as chronic viral or bacterial infection, liver disease, auto-immune status, ulcerative colitis, kidney disease and so on. [24-28] Chronic inflammation is a common cause of multiple tumors. HCC is an inflammation related carcinoma and mounting evidence suggested that persistent chronic inflammation status is associated with poor prognosis of HCC patients. [29-31] Inflammation based prognostic factors involving serum C-reactive protein and the neutrophils to lymphocyte ratio have been demonstrated as potential predictors of the CSS of liver cancer. [32-34]

ALB is produced in the liver and forms about $50 \%$ of all plasma protein. Its main function is to regulate the colloidal osmotic pressure of blood thus
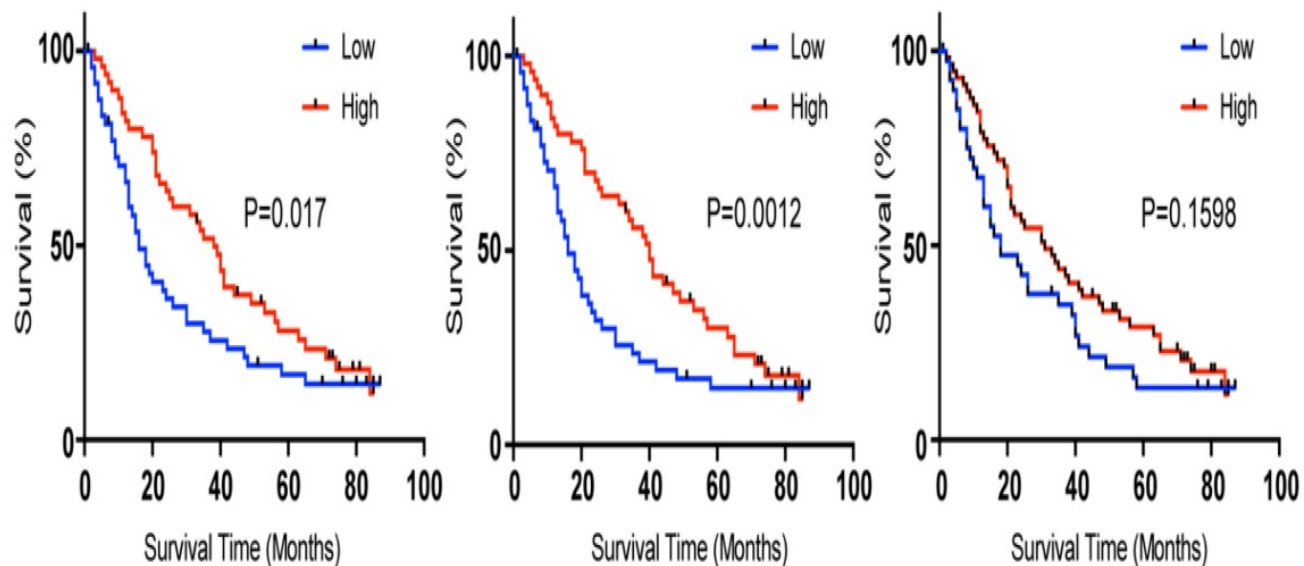

Figure 2. Kaplan-Meier analysis of CSS for ALB, GLB and AGR in the validation cohort. A: ALB B: GLB C: AGR. 
maintain the volume of whole blood. It also serves as carriers for molecules of low water solubility, such as bile salts, unconjugated bilirubin, free fatty acids and so forth. Low albumin may be caused by liver disease, nephrotic syndrome, malnutrition and malignancy. [35-37] ALB is also suggested as a potential predictor of the CSS of liver cancer. [37]

Our study found that serum GLB and ALB level were promising predictors of CSS in patients treated with HCC resection. We firstly found that high GLB level was significantly related to high AFP value, the existence of cirrhosis, major tumor size and high Edmondson grade and low ALB value was markedly associated with positive $\mathrm{HbsAg}$, high ALT and AFP level, the existence of cirrhosis, and high Edmondson grade. Then with the application of univariate analysis, GLB, ALB and AGR was suggested to be related to 5-year CSS, however after adjustment for AFP value, and the clinical characteristics with multivariate analysis, only the predicative GLB and ALB remained significant. An absolute improvement of $7.1 \%$ in 5-year CSS if $\leq 32.70 \mathrm{~g} / \mathrm{L}$ GLB level comparing to $>32.70 \mathrm{~g} / \mathrm{L}(\mathrm{P}<0.05)$. And there was a $17.6 \%$ improvement in 5-year CSS if $>40.6 \mathrm{~g} / \mathrm{L}$ ALB level rather than $\leq 40.6 \mathrm{~g} / \mathrm{L} \quad(\mathrm{P}<0.05)$. As the completed mechanisms shared by cancers and inflammation, markers of inflammatory reaction may serve as indicators of cancer diagnosis and predictors of prognosis, nevertheless, the significance of the markers was ignored to a great extent and few such markers were identified. The present study, for the first time, utilized two different cohorts to identify and confirm the cut-off of GLB and ALB, then focused on the association of serum GLB and ALB level with prognosis as well as clinicopathological parameters in HCC treated with liver section.

As a matter of fact, there exist some limitations in this study, of which, the major one would be that relevant measurements of several specific cytokines and C-reactive protein levels were missing, so consequently, we could not analyze the relationship among GLB, ALB with such inflammatory factors. Besides, this research was performed with relatively small sample, which may cause some small sample bias and limit the statistical power. Future large-scale studies involving more people with prospective designs are urgently desired.

Despite these limitations, our informative study is the first to identify the preoperative high GLB level as a prognostic risk factor for patients after curative liver resection. Furthermore, we also demonstrated $\mathrm{ALB}$ as an independent predictor for the prognosis according to our study population. Serum GLB and ALB value, moreover, can be obtained directly from routine medical laboratories, thus can act as available clinical biomarkers to predict the prognosis of HCC undergoing liver section.

\section{Supplementary Material}

Supplementary tables.

http://www.jcancer.org/v10p3494s1.pdf

\section{Acknowledgements}

This work was supported by grants from the National Key Research and Development Program of China (Grant Number: 2016YFC0905900 to B.S.); the State Key Program of National Natural Science Foundation (Grant Number: 81430062 to B.S.); Innovative Research Groups of National Natural Science Foundation (Grant Number: 81521004 to B.S.); The National Natural Science Foundation of China (Grant Number: 81702344 to W.Z.); TianQing Liver Disease Research Fund (Grant Number: TQGB20180095 to W.Z.), B.S. is Yangtze River Scholars Distinguished Professor.

\section{Ethics approval and consent to participate}

Written and publication consent was obtained from each patient, and the study was approved by Institutional Ethics Committee of the First Affiliated Hospital, Nanjing Medical University. The study was performed in accordance with the Declaration of Helsinki.

\section{Authors' contributions}

WJZ and BCS: Conceptualization, methodology, validation, investigation, writing-original draft, writing-review and editing, and visualization. WJZ, GYZY and BCS: Data curation and writing-review and editing. WJZ, HTZ and WWY: Formal analysis, investigation, and writing-review and editing. FW and YL: Investigation, resources, writing-original draft, and writing-review and editing. WJZ and BCS: Formal analysis, writing-original draft, writing-review and editing, visualization, and supervision. HTZ and GYZY: Investigation, resources, writing-original draft, and writing-review and editing. JCW, WWY and YL: Formal analysis, investigation, resources, and writing-review and editing. KPJ, ZKX and BCS: Conceptualization, methodology, software, validation, formal analysis, investigation, resources, data curation, writing-original draft, writing-review and editing, visualization, and supervision.

\section{Competing Interests}

The authors have declared that no competing interest exists. 


\section{References}

1. Ferlay J, Soerjomataram I, Dikshit R, Eser S, Mathers C, Rebelo M, et al. Cancer incidence and mortality worldwide: sources, methods and major patterns in GLOBOCAN 2012. Int J Cancer. 2015; 136: E359-86.

2. El-Serag HB. Epidemiology of viral hepatitis and hepatocellular carcinoma. Gastroenterology. 2012; 142: 1264-73 e1.

3. Utsunomiya T, Shimada M, Kudo M, Ichida T, Matsui O, Izumi N, et al. A comparison of the surgical outcomes among patients with HBV-positive, $\mathrm{HCV}$-positive, and non-B non-C hepatocellular carcinoma: a nationwide study of 11,950 patients. Ann Surg. 2015; 261: 513-20.

4. Kulik LM, Chokechanachaisakul A. Evaluation and management of hepatocellular carcinoma. Clin Liver Dis. 2015; 19: 23-43.

5. Zhang W, Jiang R, Hou J, Sun B. Clinicopathological features and prognostic factors of young patients with surgically treated liver cancer. Medicine (Baltimore). 2015; 94: e684.

6. Bray F, Ferlay J, Soerjomataram I, Siegel RL, Torre LA, Jemal A. Global cancer statistics 2018: GLOBOCAN estimates of incidence and mortality worldwide for 36 cancers in 185 countries. CA Cancer J Clin. 2018.

7. El-Serag HB, Rudolph KL. Hepatocellular carcinoma: epidemiology and molecular carcinogenesis. Gastroenterology. 2007; 132: 2557-76.

8. Hwang S, Lee YJ, Kim KH, Ahn CS, Moon DB, Ha TY, et al. The Impact of Tumor Size on Long-Term Survival Outcomes After Resection of Solitary Hepatocellular Carcinoma: Single-Institution Experience with 2558 Patients. J Gastrointest Surg. 2015; 19: 1281-90.

9. Boffa DJ, Greene FL. Reacting to changes in staging designations in the 7th edition of the AJCC staging manual. Ann Surg Oncol. 2011; 18: 1-3.

10. Nathan H, Schulick RD, Choti MA, Pawlik TM. Predictors of survival after resection of early hepatocellular carcinoma. Ann Surg. 2009; 249: 799-805.

11. Zhao HR, Jiang T, Tian YY, Gao Q, Li Z, Pan Y, et al. Angiotensin II triggers apoptosis via enhancement of NADPH oxidase-dependent oxidative stress in a dopaminergic neuronal cell line. Neurochemical research. 2015; 40: 854-63.

12. Chen J, Zhou Y, Xu Y, Zhu HY, Shi YQ. Low pretreatment serum globulin may predict favorable prognosis for gastric cancer patients. Tumour Biol. 2015.

13. Du XJ, Tang LL, Mao YP, Sun Y, Zeng MS, Kang TB, et al. The pretreatment albumin to globulin ratio has predictive value for long-term mortality in nasopharyngeal carcinoma. PLoS One. 2014; 9: e94473.

14. Maluccio M, Covey A. Recent progress in understanding, diagnosing, and treating hepatocellular carcinoma. CA: a cancer journal for clinicians. 2012; 62: 394-9.

15. Llovet JM. Focal gains of VEGFA: candidate predictors of sorafenib response in hepatocellular carcinoma. Cancer cell. 2014; 25: 560-2.

16. Hernandez-Gea V, Toffanin S, Friedman SL, Llovet JM. Role of the microenvironment in the pathogenesis and treatment of hepatocellular carcinoma. Gastroenterology. 2013; 144: 512-27.

17. Bruix J, Gores GJ, Mazzaferro V. Hepatocellular carcinoma: clinical frontiers and perspectives. Gut. 2014; 63: 844-55.

18. Johnson PJ, Berhane S, Kagebayashi C, Satomura S, Teng M, Reeves HL, et al. Assessment of liver function in patients with hepatocellular carcinoma: a new evidence-based approach-the ALBI grade. Journal of clinical oncology : official journal of the American Society of Clinical Oncology. 2015; 33: 550-8.

19. Bruix J, Reig M, Sherman M. Evidence-Based Diagnosis, Staging, and Treatment of Patients With Hepatocellular Carcinoma. Gastroenterology. 2016; 150: 835-53.

20. Greten TF, Wang XW, Korangy F. Current concepts of immune based treatments for patients with HCC: from basic science to novel treatment approaches. Gut. 2015; 64: 842-8.

21. Villanueva A, Hoshida $Y$, Battiston C, Tovar V, Sia D, Alsinet C, et al Combining clinical, pathology, and gene expression data to predict recurrence of hepatocellular carcinoma. Gastroenterology. 2011; 140: 1501-12 e2.

22. Hsu CY, Lee YH, Hsia CY, Huang YH, Su CW, Lin HC, et al. Performance status in patients with hepatocellular carcinoma: determinants, prognostic impact, and ability to improve the Barcelona Clinic Liver Cancer system. Hepatology. 2013; 57: 112-9.

23. Gelfand EW. Intravenous immune globulin in autoimmune and inflammatory diseases. The New England journal of medicine. 2012; 367: 2015-25.

24. Ooi JD, Snelgrove SL, Engel DR, Hochheiser K, Ludwig-Portugall I, Nozaki Y, et al. Endogenous foxp3(+) T-regulatory cells suppress anti-glomerular basement membrane nephritis. Kidney international. 2011; 79: 977-86.

25. Chan WL, Carrell RW, Zhou A, Read RJ. How changes in affinity of corticosteroid-binding globulin modulate free cortisol concentration. The Journal of clinical endocrinology and metabolism. 2013; 98: 3315-22.

26. Waller EK, Logan BR, Harris WA, Devine SM, Porter DL, Mineishi S, et al. Improved survival after transplantation of more donor plasmacytoid dendritic or naive T cells from unrelated-donor marrow grafts: results from BMTCTN 0201. Journal of clinical oncology : official journal of the American Society of Clinical Oncology. 2014; 32: 2365-72.

27. Simo R, Barbosa-Desongles A, Lecube A, Hernandez C, Selva DM. Potential role of tumor necrosis factor-alpha in downregulating sex hormone-binding globulin. Diabetes. 2012; 61: 372-82.

28. Stegeman BH, Helmerhorst FM, Vos HL, Rosendaal FR, Van Hylckama Vlieg A. Sex hormone-binding globulin levels are not causally related to venous thrombosis risk in women not using hormonal contraceptives. Journal of thrombosis and haemostasis : JTH. 2012; 10: 2061-7.
29. Lin MV, King LY, Chung RT. Hepatitis C virus-associated cancer. Annual review of pathology. 2015; 10: 345-70.

30. Buendia MA, Neuveut C. Hepatocellular carcinoma. Cold Spring Harbor perspectives in medicine. 2015; 5: a021444.

31. He G, Karin M. NF-kappaB and STAT3 - key players in liver inflammation and cancer. Cell research. 2011; 21: 159-68.

32. Kinoshita A, Onoda H, Imai N, Nishino H, Tajiri H. C-Reactive Protein as a Prognostic Marker in Patients with Hepatocellular Carcinoma. Hepato-gastroenterology. 2015; 62: 966-70.

33. Finkelmeier F, Canli O, Tal A, Pleli T, Trojan J, Schmidt M, et al. High levels of the soluble programmed death-ligand (sPD-L1) identify hepatocellular carcinoma patients with a poor prognosis. European journal of cancer. 2016; 59: $152-9$.

34. Lu SD, Wang YY, Peng NF, Peng YC, Zhong JH, Qin HG, et al. Preoperative Ratio of Neutrophils to Lymphocytes Predicts Postresection Survival in Selected Patients With Early or Intermediate Stage Hepatocellular Carcinoma. Medicine. 2016; 95: e2722.

35. Crawford NF, Leblanc RM. Serum albumin in 2D: a Langmuir monolayer approach. Advances in colloid and interface science. 2014; 207: 131-8.

36. Chan RW, Wong J, Chan HL, Mok TS, Lo WY, Lee V, et al. Aberrant concentrations of liver-derived plasma albumin mRNA in liver pathologies. Clinical chemistry. 2010; 56: 82-9.

37. Li T, Yu G, Guo T, Qi H, Bing Y, Xiao Y, et al. The plasma S-adenosylmethionine level is associated with the severity of hepatitis B-related liver disease. Medicine. 2015; 94: e489. 